\title{
LOCAL UNIQUENESS OF MULTI-PEAK SOLUTIONS TO A CLASS OF KIRCHHOFF EQUATIONS
}

\author{
Gongbao Li, Yahui Niu and Chang-Lin Xiang \\ Central China Normal University, School of Mathematics and Statistics \\ Wuhan, 430079, P. R. China; ligb@mail.ccnu.edu.cn \\ Central China Normal University, School of Mathematics and Statistics \\ Wuhan, 430079, P. R. China; yahuniu@163.com \\ Yangtze University, School of Information and Mathematics \\ Jingzhou 434023, P. R. China; changlin.xiang@yangtzeu.edu.cn
}

Abstract. In this paper, we consider the nonlocal Kirchhoff problem

$$
-\left(\epsilon^{2} a+\epsilon b \int_{\mathbf{R}^{3}}|\nabla u|^{2}\right) \Delta u+V(x) u=u^{p}, \quad u>0, \quad u \in H^{1}\left(\mathbf{R}^{3}\right),
$$

where $a, b>0,1<p<5$ are constants, $\epsilon>0$ is a parameter. Under some assumptions on $V(x)$, we show the local uniqueness of positive multi-peak solutions by using the local Pohozaev identity.

\section{Introduction and main results}

Let $a, b>0$ and $1<p<5$. Consider the singularly perturbed Kirchhoff problem

$$
-\left(\epsilon^{2} a+\epsilon b \int_{\mathbf{R}^{3}}|\nabla u|^{2}\right) \Delta u+V(x) u=u^{p}, \quad u>0, \quad u \in H^{1}\left(\mathbf{R}^{3}\right),
$$

where $\epsilon>0$ is a parameter, $V: \mathbf{R}^{3} \rightarrow \mathbf{R}$ is a bounded continuous function. Under some mild conditions on $V$, Luo, Peng, Wang and the last named author of the present paper [22] proved the existence of multi-peak solutions to (1.1). As a continuation of the work [22], this paper is devoted to establish a local uniqueness result for the multi-peak solutions obtained there. For physical background for equation (1.1), the readers are referred to Luo et al. [22] and the references therein.

To be precise, we first give the definition of $k$-peak solutions of Eq. (1.1) as usual.

Definition 1.1. Let $k \in \mathbf{N}, b_{j} \in \mathbf{R}^{3}, 1 \leq j \leq k$. We say that $u_{\epsilon} \in H^{1}\left(\mathbf{R}^{3}\right)$ is a $k$-peak solution of (1.1) concentrated at $\left\{b_{1}, b_{2}, \cdots, b_{k}\right\}$, if

(i) $u_{\epsilon}$ has $k$ local maximum points $x_{\epsilon}^{j} \in \mathbf{R}^{3}, j=1,2, \ldots, k$, satisfying

$$
x_{\epsilon}^{j} \rightarrow b_{j}
$$

as $\epsilon \rightarrow 0$ for each $j$;

(ii) For any given $\tau>0$, there exists $R \gg 1$, such that

$$
\left|u_{\epsilon}(x)\right| \leq \tau \quad \text { for } x \in \mathbf{R}^{3} \backslash \cup_{j=1}^{k} B_{R \epsilon}\left(x_{\epsilon}^{j}\right) ;
$$

https://doi.org/10.5186/aasfm.2020.4503

2010 Mathematics Subject Classification: Primary 35A01, 35B25, 35J20, 35J60.

Key words: Kirchhoff equations, multi-peak positive solutions, local uniqueness, local Pohozaev identity.

Corresponding author: Gongbao Li.

This work was supported by Natural Science Foundation of China (Grant No. 11771166), Hubei Key Laboratory of Mathematical Sciences and Program for Changjiang Scholars and Innovative Research Team in University \#IRT_17R46. Xiang is financially supported by NSFC (No. 11701045) and by the Yangtze Youth Fund (No. 2016cqn56). 
(iii) There exists $C>0$ such that

$$
\int_{\mathbf{R}^{3}}\left(\epsilon^{2} a\left|\nabla u_{\epsilon}\right|^{2}+u_{\epsilon}^{2}\right) \leq C \epsilon^{3} .
$$

In the simplest case $a=1, b=0$, equation (1.1) is reduced to the following perturbed Schrödinger equation:

$$
-\epsilon^{2} \Delta u+V(x) u=u^{p}, \quad u>0 \text { in } \mathbf{R}^{3},
$$

where $1<p$ is subcritical. There are extensive results on the existence of solutions to equations of type (1.2) for $\epsilon>0$ sufficiently small in the literature. We only mention for instance e.g. Floer and Weinstein [8], Oh [23, 24], del Pino and Felmer [4, 5], Gui [12], Rabinowitz [25] and the references therein.

One of our motivations of this work is from local uniqueness results for Schrödinger equations (1.2). By local uniqueness, it means that if $u_{\epsilon}^{1}, u_{\epsilon}^{2}$ are two $k$-peak solutions of equation (1.2) concentrating at the same $k$ points, then $u_{\epsilon}^{1} \equiv u_{\epsilon}^{2}$ for $\epsilon$ sufficiently small. The first result in this respect seems to be given by Glangetas [10], where the uniqueness of solutions concentrating at one point for Dirichlet problems with critical nonlinearity on bounded domains is proved. By calculating the number of single-peak solutions, Grossi [11] proved that there is only one solution concentrating at any nondegenerate critical point of $V(x)$. In fact, the uniqueness of single-bump(single-peak) solutions concentrating at some degenerate critical point of $V(x)$ is true in [11] as well. Cao and Heinz [1] proved the uniqueness of multipeak solutions to (1.2) which concentrate at nondegenerate critical points of $V(x)$ by using the topological degree, see also [10]. Recently, Deng et al. [6] proved the local uniqueness and periodicity for the solution to the prescribed scalar curvature problem

$$
-\Delta u=K(x) u^{\frac{N+2}{N-2}}, \quad u>0 \text { in } \mathbf{R}^{N}
$$

by the technique of local Pohozaev identity. Their work inspired Cao, Li and Luo [2] to establish local uniqueness of multi-peak solutions to (1.2) under some mild conditions on the potential function $V(x)$. In particular, $V(x)$ is not required to be nondegenerate at concentration points in [2]. For more local uniqueness results using local Pohozaev identities, see e.g. Guo et al. [14] for critical polyharmonic equations and Guo et al. [13] for fractional Laplacian equations.

Let us now recall briefly some recent progress on the problem (1.1). For the existence of single peak solutions to equation (1.1), we refer to e.g. He and Zou [15], Wang et al. [26], He et al. [16, 17, 18], Figueiredo and Santos Júnior [7], where variational methods have been used. We also mention the work [21] of Li et al., where Lyapunov-Schmidt reduction method is applied. Very few result for multipeak solutions of problem (1.1) exists in the literature. The first one was obtained by Luo et al. [22], where the authors found the right limiting problem for the first time, and then they established some nondegeneracy result, which allows them to apply Lyapunov-Schmidt reduction method to obtain multi- peak solutions. Another result of multi-peak solutions for Kirchhoff equations with general nonlinearity can be found in the quite recent work [19] of $\mathrm{Hu}$ and Shuai.

There are very few local uniqueness results for Kirchhoff equations. To the best of our knowledge, the only result in this respect is given by Li et al. [21], where the authors proved that single peak solutions of equation (1.1) obtained there is locally unique under some mild conditions assumed on the function $V(x)$. 
In view of the results mentioned above, a natural problem is to study the local uniqueness of the multi-peak solutions of problem (1.1) established by Luo et al. [22]. To state our main result, it is convenience to introduce the following assumptions and notations.

$\left(V_{1}\right) V \in L^{\infty}\left(\mathbf{R}^{3}\right)$ and $0<\inf _{\mathbf{R}^{3}} V \leq \sup _{\mathbf{R}^{3}} V<\infty ;$

$\left(V_{2}\right)$ There exist $m>1, \eta>0, k \in \mathbf{N}, b_{j}=\left(b_{j, 1}, b_{j, 2}, b_{j, 3}\right) \in \mathbf{R}^{3}, c_{j, i} \in \mathbf{R}$ with $c_{j, i} \neq 0$ for each $i=1,2,3$ and $j=1, \cdots, k$ such that $V \in C^{1}\left(B_{\eta}\left(b_{j}\right)\right.$ and

$$
\begin{cases}V(x)=V\left(b_{j}\right)+\sum_{i=1}^{3} c_{j, i}\left|x_{i}-b_{j, i}\right|^{m}+O\left(\left|x-b_{j}\right|^{m+1}\right), & x \in B_{\eta}\left(b_{j}\right), \\ \frac{\partial V}{\partial x_{i}}=m c_{j, i}\left|x_{i}-b_{j, i}\right|^{m-2}\left(x_{i}-b_{j, i}\right)+O\left(\left|x-b_{j}\right|^{m}\right), & x \in B_{\eta}\left(b_{j}\right),\end{cases}
$$

where $x=\left(x_{1}, x_{2}, x_{3}\right) \in \mathbf{R}^{3}$.

Denote

$$
\begin{aligned}
\langle u, v\rangle_{\epsilon} & =\int_{\mathbf{R}^{3}}\left(\epsilon^{2} a \nabla u \cdot \nabla v+V(x) u v\right) \quad \text { and } \quad\|u\|_{\epsilon}^{2}=\langle u, u\rangle_{\epsilon} \\
H_{\epsilon} & =\left\{u \in H^{1}\left(\mathbf{R}^{3}\right):\|u\|_{\epsilon} \equiv\langle u, u\rangle_{\epsilon}^{1 / 2}<\infty\right\} .
\end{aligned}
$$

The energy functional corresponding to Eq. (1.1) is

$$
I_{\epsilon}(u)=\frac{1}{2}\|u\|_{\epsilon}^{2}+\frac{b \epsilon}{4}\left(\int_{\mathbf{R}^{3}}|\nabla u|^{2}\right)^{2}-\frac{1}{p+1} \int_{\mathbf{R}^{3}} u_{+}^{p+1}
$$

for $u \in H_{\epsilon}$, where $u_{+}=\max \{u, 0\}$.

We call $u \in H_{\epsilon}$ a (weak) solution to equation (1.1) if for any $\psi \in H_{\epsilon}$, it holds that

$$
\langle u, \psi\rangle_{\epsilon}+b \epsilon \int_{\mathbf{R}^{3}}|\nabla u|^{2} \int_{\mathbf{R}^{3}} \nabla u \cdot \nabla \psi=\int_{\mathbf{R}^{3}} u^{p} \psi
$$

Recall that it was proven in [22, Proposition 2.3] that the limiting problem of Eq. (1.1) is given by the following system

$$
\begin{cases}-\left(a+b \sum_{j=1}^{k} \int\left|\nabla U^{j}\right|^{2}\right) \Delta U^{i}+V\left(b_{i}\right) U^{i}=\left(U^{i}\right)^{p} & \text { in } \mathbf{R}^{3} \\ U^{i}>0 & \text { in } \mathbf{R}^{3} \\ U^{i} \in H^{1}\left(\mathbf{R}^{3}\right) . & \end{cases}
$$

Hereafter, we denote by $\left(U^{1}, \cdots, U^{k}\right)$ the unique positive radial solutions to the above system (see [22]). Keep in mind that each $U^{i}$ is also nondegenerate.

Our main result is as follows:

Theorem 1.2. Assume that $V(x)$ satisfies $\left(V_{1}\right)-\left(V_{2}\right)$. If $u_{\epsilon}^{(1)}, u_{\epsilon}^{(2)}$ are two $k$-peak solutions concentrating at the set of $k$ different points $\left\{b_{1}, \cdots, b_{k}\right\} \subset \mathbf{R}^{3}$ appeared in the condition $\left(V_{2}\right)$, then for $\epsilon$ sufficiently small, we have

$$
u_{\epsilon}^{(1)} \equiv u_{\epsilon}^{(2)}
$$

and $u_{\epsilon}^{(j)}(j=1,2)$ is of the form

$$
u_{\epsilon}^{(j)}=\sum_{i=1}^{k} U^{i}\left(\left(x-x_{\epsilon}^{i}\right) / \epsilon\right)+\varphi_{\epsilon}(x)
$$

with $x_{\epsilon}^{i} \in \mathbf{R}^{3}, \varphi_{\epsilon} \in H_{\epsilon}$ satisfying

$$
\left|x_{\epsilon}^{i}-b_{i}\right|=o(\epsilon),\left\|\varphi_{\epsilon}\right\|_{\epsilon}=O\left(\epsilon^{3 / 2+m}\right)
$$

for $i=1, \cdots, k$. 
We first explain a characteristic of this multi-peak problem, which is different from the case of Schrödinger equation. To construct multi-peak solutions to the Schrödinger equation (1.2), it is very important to understand the limiting equation as $\epsilon \rightarrow 0$, which is known as the unperturbed Schrödinger equation $-\Delta w+w=w^{q}$. Denote by $w_{i}$ the unique (see [20]) positive radial solution to equation

$$
-\Delta w_{i}+V\left(b_{i}\right) w_{i}=w_{i}^{q} \quad \text { in } \mathbf{R}^{n} .
$$

Then, to construct a $k$-peak solution to Eq. (1.2) concentrated at $\left\{b_{1}, \ldots, b_{k}\right\}$, natural candidates are functions of the form $u_{\epsilon}=\sum_{i=1}^{k} w_{i}\left(\left(x-x_{\epsilon}^{i}\right) / \epsilon\right)+\varphi_{\epsilon}$, where $x_{\epsilon}^{i} \rightarrow b_{i}$ and $\varphi_{\epsilon}$ should be appropriately chosen such that $u_{\epsilon}$ is indeed a solution to equation (1.2). Accordingly, to construct multi-peak solutions to (1.1), a natural idea is that the building block of the multi-peak solution should be the unique positive radial solution $w_{i}$ of the unperturbed Kirchhoff equation

$$
-\left(a+b \int_{\mathbf{R}^{3}}|\nabla u|^{2}\right) \Delta u+V\left(b_{i}\right) u=u^{p}, \quad u>0 \text { in } \mathbf{R}^{3} .
$$

while the fact is that this idea only works for the single-peak solution, as can be seen in Li et al. [21]. That is, there is no multi-peak solutions for (1.1) of the form $u_{\epsilon}=\sum_{i=1}^{k} w^{i}\left(\left(x-x_{\epsilon}^{i}\right) / \epsilon\right)+\varphi_{\epsilon}$, (see [22, Proposition 2.2]). Furthermore, during their proof of that the blow-up point $b_{j}$ must be critical points of $V(x)$, they found that the right limiting equation of (1.1) is in fact a system of partial differential equations (see [22, Proposition 2.3]).

We mainly use the method in [2] to prove the main result. However, the nonlocal term $\left(\int_{\mathbf{R}^{3}}|\nabla u|^{2}\right) \Delta u$ causes more technical difficulties than Schrödinger equation (1.2). Moreover, as we consider the multi-peak solution of (1.1), on the one hand, there are many crosses between bubbles, and on the other hand, the limiting equation of (1.1) is a system of partial differential equations (see (1.4)), which is different from the case of single-peak solution and Schrödinger equation (1.2), the difference of these two aspects also makes the estimate more complex.

Now, we give the main idea of the proof of Theorem 1.2. We will follow the idea of Cao, Li and Luo [2]. More precisely, if $u_{\epsilon}^{(1)}, u_{\epsilon}^{(2)}$ are two distinct solutions as stated in Theorem 1.2, according to Proposition 2.1 in this paper, they have the same form; We firstly get the improved estimate of $\left|x_{\epsilon}^{i}-b_{i}\right|$ and $\left\|\varphi_{\epsilon}\right\|_{\epsilon}$, then for the function $\xi_{\epsilon}=\left(u_{\epsilon}^{(1)}-u_{\epsilon}^{(2)}\right) /\left\|u_{\epsilon}^{(1)}-u_{\epsilon}^{(2)}\right\|_{L^{\infty}\left(\mathbf{R}^{3}\right)}$, which obviously satisfies $\left\|\xi_{\epsilon}\right\|_{L^{\infty}\left(\mathbf{R}^{3}\right)}=1$, we show that $\left\|\xi_{\epsilon}\right\|_{L^{\infty}\left(\mathbf{R}^{3}\right)} \rightarrow 0$ as $\epsilon \rightarrow 0$, which gives a contradiction, and thus the uniqueness is proved. During the above process, an important tool is a type of local Pohozaev identity (see (2.8) below) from Deng, Lin and Yan [6], which helps us to get the rate of the maximum points $x_{\epsilon}^{i}$ tending to $b_{i}$ as $\epsilon \rightarrow 0$. For the estimate of $\left\|\varphi_{\epsilon}\right\|_{\epsilon}$, we will derive from the nondegeneracy result of Luo et al. [22]. We will need more careful analysis in the procedure due to the presence of the nonlocal term $\left(\int_{\mathbf{R}^{3}}|\nabla u|^{2}\right) \Delta u$, which leads to the local Pohozaev identity more complicated than the case of the Schrödinger equation (1.2).

Our notations are standard. We write $\int u$ to denote Lebesgue integral of $u$ over $\mathbf{R}^{3}$, unless otherwise stated, and $\|u\|_{p}=\left(\int_{\mathbf{R}^{3}} u^{p}\right)^{1 / p}$. We use $B_{R}(x)$ (and $\left.\bar{B}_{R}(x)\right)$ to denote open (and close) balls in $\mathbf{R}^{3}$ centered at $x$ with radius $R$. We will use $C$ and $C_{j}(j \in \mathbf{N})$ to denote various positive constants, and $O(t), o(t), o_{t}(1)$ to mean $|O(t)| \leq C|t|, o(t) / t \rightarrow 0$ and $o_{t}(1) \rightarrow 0$ as $t \rightarrow 0$, respectively.

The paper is organized as follows. In section 2 we give some preliminary estimates. In section 3 we prove the local uniqueness results. 


\section{Preliminaries}

According to [22, Proposition 2.3], the form of $k$-peak solutions to (1.1) are given by the following:

Proposition 2.1. Let $k \in \mathbf{N},\left\{b_{1}, b_{2}, \ldots, b_{k}\right\} \subset \mathbf{R}^{3}$ and $u_{\epsilon}$ be a $k$-peak solution of Eq. (1.1) defined as in the Definition 1.1, with local maximum points at $x_{\epsilon}^{i}$. Then, for $\epsilon>0$ sufficiently small, $u_{\epsilon}$ is of the form

$$
u_{\epsilon}(x)=\sum_{i=1}^{k} U^{i}\left(\left(x-x_{\epsilon}^{i}\right) / \epsilon\right)+\varphi_{\epsilon}(x)
$$

with

$$
\left|x_{\epsilon}^{i}-b_{i}\right|=o(1), \quad\left\|\varphi_{\epsilon}\right\|_{\epsilon}=o\left(\epsilon^{\frac{3}{2}}\right) .
$$

For simplicity in the below, we introduce the notation

$$
w_{\epsilon, y}(x)=w((x-y) / \epsilon) .
$$

for any function $w$. The estimates given by (2.2) is not sufficient for later use. Our aim in this section is to prove the following proposition.

Proposition 2.2. Assume that $V$ satisfies $\left(V_{1}\right)$ and $\left(V_{2}\right)$. Let $k \in \mathbf{N},\left\{b_{1}, \ldots, b_{k}\right\}$ $\subset \mathbf{R}^{3}$ and $u_{\epsilon}$ be a $k$-peak solution of Eq. (1.1) defined as in the Definition 1.1, with local maximum points at $x_{\epsilon}^{i}$. Suppose that

$$
u_{\epsilon}(x)=\sum_{i=1}^{k} U_{\epsilon, x_{\epsilon}^{i}}^{i}+\varphi_{\epsilon}(x) .
$$

Then

$$
\left|x_{\epsilon}^{i}-b_{i}\right|=o(\epsilon)
$$

for each $i, 1 \leq i \leq k$, and

$$
\left\|\varphi_{\epsilon}\right\|_{\epsilon}=O\left(\epsilon^{3 / 2+m}\right) \text {. }
$$

Before proving the above proposition, we first collect some useful facts. Note that since the unique positive solution of equation $-\Delta u+V\left(b_{i}\right) u=u^{p}$ decays exponentially at infinity (see [9]), we infer that

$$
\max _{1 \leq i \leq k}\left(U^{i}(x)+\left|\nabla U^{i}(x)\right|\right)=O\left(e^{-\sigma|x|}\right)
$$

for some $\sigma>0$. Let $0<\delta<\min \left\{\left|b_{i}-b_{j}\right| / 4: i \neq j\right\}$ and denote

$$
D_{\delta}=\bar{B}_{\delta}\left(b_{1}\right) \times \cdots \times \bar{B}_{\delta}\left(b_{k}\right) .
$$

Note that if $\left(x_{\epsilon}^{1}, \cdots, x_{\epsilon}^{k}\right) \in D_{\delta}$, then $\left|x_{\epsilon}^{i}-x_{\epsilon}^{j}\right| \geq\left|b_{i}-b_{j}\right| / 2 \geq 2 \delta$ for $i \neq j$, which implies by (2.5) that

$$
\int \nabla U_{\epsilon, x_{\epsilon}^{i}}^{i} \cdot \nabla U_{\epsilon, x_{\epsilon}^{j}}^{j}+\left(U_{\epsilon, x_{\epsilon}^{i}}^{i}\right)^{r}\left(U_{\epsilon, x_{\epsilon}^{j}}^{j}\right)^{s}=O\left(e^{-\gamma / \epsilon}\right) \quad \text { if } i \neq j
$$

for any given $r, s>0$, where $\gamma>0$ is a constant.

We recall an inequality: For any $2 \leq q \leq 6$ there exists a constant $C>0$ depending only on $n, a$ and $q$ but independent of $\epsilon$, such that

$$
\|\varphi\|_{L^{q}\left(\mathbf{R}^{3}\right)} \leq C \epsilon^{\frac{3}{q}-\frac{3}{2}}\|\varphi\|_{\epsilon}
$$

holds for all $\varphi \in H_{\epsilon}$. For a proof, see e.g. (3.6) of Li et al. [21].

The crucial Pohozaev type identity we will use is as follows: 
Proposition 2.3. Let $u$ be a positive solution to Eq. (1.1). Let $\Omega$ be a bounded smooth domain in $\mathbf{R}^{3}$ such that $V \in C^{1}(\Omega)$ holds. Then

$$
\begin{aligned}
\int_{\Omega} \frac{\partial V}{\partial x_{\alpha}} u^{2}= & \left(\epsilon^{2} a+\epsilon b \int|\nabla u|^{2}\right) \int_{\partial \Omega}\left(|\nabla u|^{2} \nu_{\alpha}-2 \frac{\partial u}{\partial \nu} \frac{\partial u}{\partial x_{\alpha}}\right) \\
& +\int_{\partial \Omega} V u^{2} \nu_{\alpha}-\frac{2}{p+1} \int_{\partial \Omega} u^{p+1} \nu_{\alpha}
\end{aligned}
$$

for each $\alpha=1,2,3$, where $\nu=\left(\nu_{1}, \nu_{2}, \nu_{3}\right)$ is the unit outward normal of $\partial \Omega$.

Proposition 2.3 can be proved by multiplying both sides of equation (1.1) by $\partial_{x_{\alpha}} u$ for each $1 \leq \alpha \leq 3$ and then integrating by parts. We omit the proof, see Cao, Li and Luo [2, Proposition 2.3] for the details.

Proof of Proposition 2.2. For simplicity, write $\varphi=\varphi_{\epsilon}$ in the following. Denote $X_{\epsilon}=\left(x_{\epsilon}^{1}, \cdots, x_{\epsilon}^{k}\right), \quad G_{\epsilon, X_{\epsilon}}=\sum_{i=1}^{k} U_{\epsilon, x_{\epsilon}^{i}}^{i}$. Let

$$
\begin{aligned}
\mathcal{L}_{\epsilon} \varphi= & -\left(\epsilon^{2} a+\epsilon b \int\left|\nabla G_{\epsilon, X_{\epsilon}}\right|^{2}\right) \Delta \varphi \\
& -2 \epsilon b\left(\int \nabla G_{\epsilon, X_{\epsilon}} \cdot \nabla \varphi\right) \Delta G_{\epsilon, X_{\epsilon}}+V(x) \varphi-p G_{\epsilon, X_{\epsilon}}^{p-1} \varphi,
\end{aligned}
$$

for $\varphi \in E_{\epsilon, X_{\epsilon}}$, where

$$
E_{\epsilon, X_{\epsilon}}=\left\{u \in H_{\epsilon}:\left\langle u, \partial_{x_{\epsilon, j}^{i}} U_{\epsilon, x_{\epsilon}^{i}}^{i}\right\rangle=0 \text { for } i=1, \cdots, k, j=1,2,3\right\} .
$$

By Proposition 3.4 of [22], there exist $\epsilon, \delta>0$ small enough, and $\rho>0$ such that

$$
\rho\|\varphi\|_{\epsilon} \leq\left\|\mathcal{L}_{\epsilon} \varphi\right\|_{\epsilon}, \quad \varphi \in E_{\epsilon, X_{\epsilon}} .
$$

Now we compute $\mathcal{L}_{\epsilon} \varphi$. By the equation (1.4) of $U^{i}$, we have

$$
-\left(\epsilon^{2} a+\epsilon b \sum_{j=1}^{k} \int\left|\nabla U_{\epsilon, x_{\epsilon}^{j}}^{j}\right|^{2}\right) \Delta U_{\epsilon, x_{\epsilon}^{i}}^{i}+V\left(b_{i}\right) U_{\epsilon, x_{\epsilon}^{i}}^{i}=\left(U_{\epsilon, x_{\epsilon}^{i}}^{i}\right)^{p} \quad \text { in } \mathbf{R}^{3} .
$$

Summing over $i$ on both sides of the equation, we have

$$
-\left(\epsilon^{2} a+\epsilon b \sum_{j=1}^{k} \int\left|\nabla U_{\epsilon, x_{\epsilon}^{j}}^{j}\right|^{2}\right) \Delta G_{\epsilon, X_{\epsilon}}+\sum_{i=1}^{k} V\left(b_{i}\right) U_{\epsilon, x_{\epsilon}^{i}}^{i}=\sum_{i=1}^{k}\left(U_{\epsilon, x_{\epsilon}^{i}}^{i}\right)^{p} \text { in } \mathbf{R}^{3} .
$$

As $u_{\epsilon}$ is a solution of equation (1.1), we have

$$
-\left(\epsilon^{2} a+\epsilon b \int\left|\nabla G_{\epsilon, X_{\epsilon}}+\nabla \varphi\right|^{2}\right) \Delta\left(G_{\epsilon, X_{\epsilon}}+\varphi\right)+V(x)\left(G_{\epsilon, X_{\epsilon}}+\varphi\right)=\left(G_{\epsilon, X_{\epsilon}}+\varphi\right)^{p}
$$

As a result, $\varphi$ must satisfy

$$
\mathcal{L}_{\epsilon} \varphi=\sum_{j=1}^{3} r_{j}+\sum_{j=1}^{3} f_{j}
$$

with

$$
\begin{aligned}
& r_{1}=\epsilon b\left(\int|\nabla \varphi|^{2}\right) \Delta u_{\epsilon}, \quad r_{2}=2 \epsilon b\left(\int \nabla G_{\epsilon, X_{\epsilon}} \cdot \nabla \varphi\right) \Delta \varphi, \\
& r_{3}=\epsilon b\left(\int\left|\nabla G_{\epsilon, X_{\epsilon}}\right|^{2}-\sum_{i=1}^{k} \int\left|\nabla U_{\epsilon, x_{\epsilon}^{i}}^{i}\right|^{2}\right) \Delta G_{\epsilon, X_{\epsilon}},
\end{aligned}
$$




$$
\begin{aligned}
f_{1} & =\left(\sum_{i=1}^{k} U_{\epsilon, x_{\epsilon}^{i}}^{i}+\varphi\right)^{p}-\left(\sum_{i=1}^{k} U_{\epsilon, x_{\epsilon}^{i}}^{i}\right)^{p}-p\left(\sum_{i=1}^{k} U_{\epsilon, x_{\epsilon}^{i}}^{i}\right)^{p-1} \varphi \\
f_{2} & =\left(\sum_{i=1}^{k} U_{\epsilon, x_{\epsilon}^{i}}^{i}\right)^{p}-\sum_{i=1}^{k}\left(U_{\epsilon, x_{\epsilon}^{i}}^{i}\right)^{p}, \\
f_{3} & =\sum_{i=1}^{k}\left(V\left(b_{i}\right)-V(x)\right) U_{\epsilon, x_{\epsilon}^{i}}^{i} .
\end{aligned}
$$

Hence $^{1}$

$$
\rho\|\varphi\|_{\epsilon}^{2} \leq\left\langle\mathcal{L}_{\epsilon} \varphi, \varphi\right\rangle=\sum_{j=1}^{3} \int r_{j} \varphi+\sum_{j=1}^{3} \int f_{j} \varphi .
$$

By the fact that

$$
\int|\nabla \varphi|^{2}=O\left(\epsilon^{-2}\|\varphi\|_{\epsilon}^{2}\right)
$$

we have

$$
\begin{gathered}
\int r_{1} \varphi=\epsilon b\left(\int|\nabla \varphi|^{2}\right) \int \nabla\left(G_{\epsilon, X_{\epsilon}}+\varphi\right) \cdot \nabla \varphi \\
=O\left(\epsilon^{-1}\|\varphi\|_{\epsilon}^{2}\right)\left(\epsilon^{-\frac{1}{2}}\|\varphi\|_{\epsilon}+\epsilon^{-2}\|\varphi\|_{\epsilon}^{2}\right)=O\left(\epsilon^{-\frac{3}{2}}\|\varphi\|_{\epsilon}^{3}+\epsilon^{-3}\|\varphi\|_{\epsilon}^{4}\right), \\
\int r_{2} \varphi=2 \epsilon b\left(\int \nabla G_{\epsilon, X_{\epsilon}} \cdot \nabla \varphi\right) \int|\nabla \varphi|^{2}=O\left(\epsilon \cdot \epsilon^{\frac{1}{2}} \cdot \epsilon^{-1}\|\varphi\|_{\epsilon} \cdot \epsilon^{-2}\|\varphi\|_{\epsilon}^{2}\right)=O\left(\epsilon^{-\frac{3}{2}}\|\varphi\|_{\epsilon}^{3}\right)
\end{gathered}
$$

and

$$
\begin{aligned}
\int r_{3} \varphi & =\epsilon b\left(\int\left|\nabla G_{\epsilon, X_{\epsilon}}\right|^{2}-\sum_{i=1}^{k} \int\left|\nabla U_{\epsilon, x_{\epsilon}^{i}}^{i}\right|^{2}\right) \int \nabla G_{\epsilon, X_{\epsilon}} \nabla \varphi \\
& =\epsilon b \int \sum_{i \neq j} \nabla U_{\epsilon, x_{\epsilon}^{i}}^{i} \nabla U_{\epsilon, x_{\epsilon}^{j}}^{j} \int \nabla G_{\epsilon, X_{\epsilon}} \nabla \varphi=O\left(e^{-\frac{\gamma}{\epsilon}}\|\varphi\|_{\epsilon}\right),
\end{aligned}
$$

where $\gamma>0$ is a constant.

Similar to the proof of (5.14) and (5.15) in [2], we have, for $j=1$ and $j=3$,

$$
\int f_{j} \varphi=\epsilon^{\frac{3}{2}} O\left(\epsilon^{m}+\left|x_{\epsilon}^{i}-b_{i}\right|^{m}\right)\|\varphi\|_{\epsilon} .
$$

Since each $U^{i}$ decays exponentially, we also derive that

$$
\int f_{2} \varphi=O\left(\epsilon^{\gamma}\right)\|\varphi\|_{\epsilon}
$$

for any $\gamma>0$. Combining the above estimate with (2.10), we have

$$
\|\varphi\|_{\epsilon}=O\left(\epsilon^{-\frac{3}{2}}\|\varphi\|_{\epsilon}^{2}+\epsilon^{-3}\|\varphi\|_{\epsilon}^{3}\right)+\epsilon^{\frac{3}{2}} O\left(\epsilon^{m}+\left|x_{\epsilon}^{i}-b_{i}\right|^{m}\right) .
$$

As a result,

$$
\|\varphi\|_{\epsilon}=\epsilon^{\frac{3}{2}} O\left(\epsilon^{m}+\left|x_{\epsilon}^{i}-b_{i}\right|^{m}\right) .
$$

\footnotetext{
${ }^{1}$ Here we apply an additional property that $\rho\|\varphi\|_{\epsilon}^{2} \leq\left\langle\mathcal{L}_{\epsilon} \varphi, \varphi\right\rangle$ holds for $\varphi \in E_{\epsilon, X_{\epsilon}}$. As the proof is standard (see e.g. [3]), we omit the details.
} 
Next we prove $(2.3)$ for fix $i \in\{1, \cdots, k\}$. It suffices to choose $i$ such that

$$
\left|x_{\epsilon}^{i}-b_{i}\right|=\max \left\{\left|x_{\epsilon}^{j}-b_{j}\right|: 1 \leq j \leq k\right\} .
$$

Applying the Pohozaev-type identity (2.8) to $u=u_{\epsilon}$ with $\Omega=B_{d}\left(x_{\epsilon}^{i}\right)$, where $0<$ $d<\delta$ is chosen in such a way (see [2, Lemma 4.5] for details) that

$$
\int_{\partial B_{d}\left(x_{\epsilon}^{i}\right)}\left(\epsilon^{2}|\nabla \varphi|^{2}+V(x)|\varphi|^{2}+|\varphi|^{p}\right)=O\left(\|\varphi\|_{\epsilon}^{2}+\int|\varphi|^{p}\right)
$$

for any $\varphi \in H_{\epsilon}$. We have

$$
\begin{aligned}
\int_{B_{d}\left(x_{\epsilon}^{i}\right)} \frac{\partial V(x)}{\partial x_{\alpha}} u_{\epsilon}^{2}= & \left(\epsilon^{2} a+\epsilon b \int\left|\nabla u_{\epsilon}\right|^{2}\right) \int_{\partial B_{d}\left(x_{\epsilon}^{i}\right)}\left(\left|\nabla u_{\epsilon}\right|^{2} \nu_{\alpha}-2 \frac{\partial u_{\epsilon}}{\partial \nu} \frac{\partial u_{\epsilon}}{\partial x_{\alpha}}\right) \\
& +\int_{\partial B_{d}\left(x_{\epsilon}^{i}\right)} V(x) u_{\epsilon}^{2} \nu_{\alpha}-\frac{2}{p+1} \int_{\partial B_{d}\left(x_{\epsilon}^{i}\right)} u_{\epsilon}^{p+1} \nu_{\alpha} .
\end{aligned}
$$

We estimate each side of (2.14) as follows. By Proposition 2.1, we know $\|\varphi\|_{\epsilon}=o\left(\epsilon^{\frac{3}{2}}\right)$. Thus, a straightforward computation gives

$$
\left\|u_{\epsilon}\right\|_{\epsilon}=O\left(\epsilon^{\frac{3}{2}}\right)
$$

So

$$
\epsilon^{2} a+\epsilon b \int\left|\nabla u_{\epsilon}\right|^{2}=O\left(\epsilon^{2}\right)
$$

By an elementary inequality, we have

$$
\left|\nabla u_{\epsilon}\right|^{2} \leq C \sum_{i=1}^{k}\left|\nabla U_{\epsilon, x_{\epsilon}^{i}}^{i}\right|^{2}+C|\nabla \varphi|^{2} .
$$

From (2.5), we know

$$
\int_{\partial B_{d}\left(x_{\epsilon}^{i}\right)} \sum_{i=1}^{k}\left|\nabla U_{\epsilon, x_{\epsilon}^{i}}^{i}\right|^{2}=O\left(e^{-\frac{\lambda}{\epsilon}}\right)
$$

holds for some $\lambda>0$ independent of $\epsilon$. As a result,

$$
\left(\epsilon^{2} a+\epsilon b \int\left|\nabla u_{\epsilon}\right|^{2}\right) \int_{\partial B_{d}\left(x_{\epsilon}^{i}\right)}\left(\left|\nabla u_{\epsilon}\right|^{2} \nu_{\alpha}-2 \frac{\partial u_{\epsilon}}{\partial \nu} \frac{\partial u_{\epsilon}}{\partial x_{\alpha}}\right)=O\left(\epsilon^{\gamma}+\|\varphi\|_{\epsilon}^{2}\right)
$$

for any $\gamma>0$. Using similar arguments and choosing a suitable $d$ if necessary, we also derive

$$
\int_{\partial B_{d}\left(x_{\epsilon}^{i}\right)} V(x) u_{\epsilon}^{2} \nu_{\alpha}=O\left(\epsilon^{\gamma}+\|\varphi\|_{\epsilon}^{2}\right)
$$

since $V(x)$ is bounded. Furthermore, from (2.7) and (2.13), we have

$$
\int_{\partial B_{d}\left(x_{\epsilon}^{i}\right)} u_{\epsilon}^{p+1} \nu_{\alpha}=O\left(\epsilon^{\gamma}+\left(\epsilon^{\frac{3}{p+1}-\frac{3}{2}}\|\varphi\|_{\epsilon}\right)^{p+1}\right)=O\left(\epsilon^{\gamma}+\|\varphi\|_{\epsilon}^{2}\right) .
$$

Combing the above estimate and (2.14), we obtain

$$
\int_{B_{d}\left(x_{\epsilon}^{i}\right)} \frac{\partial V}{\partial x_{\alpha}} u_{\epsilon}^{2}=O\left(\epsilon^{\gamma}+\|\varphi\|_{\epsilon}^{2}\right)
$$

for any $\gamma>0$. 
Next we estimate the left hand side of (2.18). Substitute the form of $u_{\epsilon}$ into the integral, and recall (2.6). We obtain

$$
\int_{B_{d}\left(x_{\epsilon}^{i}\right)} \frac{\partial V}{\partial x_{\alpha}} u_{\epsilon}^{2}=\int_{B_{d}\left(x_{\epsilon}^{i}\right)} \frac{\partial V}{\partial x_{\alpha}}\left(U_{\epsilon, x_{\epsilon}^{i}}^{i}\right)^{2}+2 \int_{B_{d}\left(x_{\epsilon}^{i}\right)} \frac{\partial V}{\partial x_{\alpha}} U_{\epsilon, x_{\epsilon}^{i}}^{i} \varphi+O\left(\|\varphi\|_{\epsilon}^{2}+\epsilon^{\gamma}\right)
$$

for any given constant $\gamma>0$. By the assumption $\left(V_{2}\right)$ and Hölder's inequality, there holds

$$
\int_{B_{d}\left(x_{\epsilon}^{i}\right)} \frac{\partial V}{\partial x_{\alpha}} U_{\epsilon, x_{\epsilon}^{i}}^{i} \varphi=\epsilon^{\frac{3}{2}} O\left(\epsilon^{m-1}+\left|x_{\epsilon}^{i}-b_{i}\right|^{m-1}\right)\|\varphi\|_{\epsilon}
$$

Applying the assumption $\left(V_{2}\right)$, we have

$$
\begin{aligned}
& \int_{B_{d}\left(x_{\epsilon}^{i}\right)} \frac{\partial V}{\partial x_{\alpha}}\left(U_{\epsilon, x_{\epsilon}^{i}}^{i}\right)^{2} \\
& =m c_{i, \alpha} \int_{B_{d}\left(x_{\epsilon}^{i}\right)}\left|x_{\alpha}-b_{i, \alpha}\right|^{m-2}\left(x_{\alpha}-b_{i, \alpha}\right)\left(U_{\epsilon, x_{\epsilon}^{i}}^{i}\right)^{2} \\
& \quad+O\left(\int_{B_{d}\left(x_{\epsilon}^{i}\right)}\left|x_{\alpha}-b_{i, \alpha}\right|^{m}\left(U_{\epsilon, x_{\epsilon}^{i}}^{i}\right)^{2}\right) \\
& =m c_{i, \alpha} \epsilon^{3} \int_{B_{\frac{d}{\epsilon}}(0)}\left|\epsilon x_{\alpha}+x_{\epsilon, \alpha}^{i}-b_{i, \alpha}\right|^{m-2}\left(\epsilon x_{\alpha}+x_{\epsilon, \alpha}^{i}-b_{i, \alpha}\right)\left(U^{i}\right)^{2} \\
& \quad+\epsilon^{3} O\left(\epsilon^{m}+\left|x_{\epsilon}^{i}-b_{i}\right|^{m}\right),
\end{aligned}
$$

where $x_{\epsilon, \alpha}^{i}$ is the $\alpha$ th exponent of $x_{\epsilon}^{i}$.

Therefore, combining (2.18)-(2.21) and (2.12) yields

$$
\int_{B_{\frac{d}{\epsilon}}(0)}\left|\epsilon x_{\alpha}+x_{\epsilon, \alpha}^{i}-b_{i, \alpha}\right|^{m-2}\left(\epsilon x_{\alpha}+x_{\epsilon, \alpha}^{i}-b_{i, \alpha}\right)\left(U^{i}(x)\right)^{2}=O\left(\epsilon^{m}+\left|x_{\epsilon}^{i}-b_{i}\right|^{m}\right) .
$$

Note that, for $a, b \in \mathbf{R}, m>1, m^{*}=\min \{m, 2\}$, we have the following inequality

$$
|| a+\left.b\right|^{m}-|a|^{m}-m|a|^{m-2} a b \mid \leq C\left(|a|^{m-m^{*}}|b|^{m^{*}}+b^{m}\right)
$$

where the constant $C$ is independent of $a, b$.

Applying (2.23) to $a=\epsilon x_{\alpha}+x_{\epsilon, \alpha}^{i}-b_{i, \alpha}$ and $b=-\epsilon x_{\alpha}$, we have

$$
\begin{aligned}
& \left|x_{\epsilon, \alpha}^{i}-b_{i, \alpha}\right|\left|\int_{B_{\frac{d}{\epsilon}}(0)}\right| \epsilon x_{\alpha}+x_{\epsilon, \alpha}^{i}-\left.b_{i, \alpha}\right|^{m-2}\left(\epsilon x_{\alpha}+x_{\epsilon, \alpha}^{i}-b_{i, \alpha}\right)\left(U^{i}(x)\right)^{2} \mid \\
& \geq \frac{1}{m}\left|x_{\epsilon, \alpha}^{i}-b_{i, \alpha}\right|^{m} \int_{B_{\frac{d}{\epsilon}}(0)}\left(U^{i}(x)\right)^{2} \\
& \quad-\frac{C}{m} \int_{B_{\frac{d}{\epsilon}}(0)}\left(\left|\epsilon x_{\alpha}\right|^{m}+\left|\epsilon x_{\alpha}\right|^{m^{*}}\left|x_{\epsilon, \alpha}^{i}-b_{i, \alpha}\right|^{m-m^{*}}\right)\left(U^{i}(x)\right)^{2} .
\end{aligned}
$$

Take $\alpha=i_{0} \in\{1,2,3\}$ such that $\left|x_{\epsilon, i_{0}}^{i}-b_{i, i_{0}}\right| \geq \frac{\left|x_{\epsilon}^{i}-b_{i}\right|}{\sqrt{3}}$. Note also that $\left|x_{\epsilon, i_{0}}^{i}-b_{i, i_{0}}\right| \leq$ $\left|x_{\epsilon}^{i}-b_{i}\right|$. Thus, combining with (2.22) and (2.24), we have

$$
\begin{aligned}
\left|x_{\epsilon}^{i}-b_{i}\right|^{m} & =\left|x_{\epsilon}^{i}-b_{i}\right| O\left(\epsilon^{m}+\left|x_{\epsilon}^{i}-b_{i}\right|^{m}\right)+O\left(\epsilon^{m}+\left|x_{\epsilon}^{i}-b_{i}\right|^{m-m^{*}} \epsilon^{m^{*}}\right) \\
& =\left|x_{\epsilon}^{i}-b_{i}\right| O\left(\epsilon^{m}+\left|x_{\epsilon}^{i}-b_{i}\right|^{m}\right)+O\left(\epsilon^{m}\right)+\frac{1}{2}\left|x_{\epsilon}^{i}-b_{i}\right|^{m}
\end{aligned}
$$


which implies

Then, from (2.22), we have

$$
\left|x_{\epsilon}^{i}-b_{i}\right|=O(\epsilon)
$$

$$
\int_{B_{\frac{d}{\epsilon}}(0)}\left|x_{\alpha}+\frac{x_{\epsilon, \alpha}^{i}-b_{i, \alpha}}{\epsilon}\right|^{m-2}\left(x_{\alpha}+\frac{x_{\epsilon, \alpha}^{i}-b_{i, \alpha}}{\epsilon}\right)\left(U^{i}(x)\right)^{2}=O(\epsilon) .
$$

From $\left|x_{\epsilon}^{i}-b_{i}\right|=O(\epsilon)$, we suppose $\frac{x_{\epsilon}^{i}-b_{i}}{\epsilon} \rightarrow t$ as $\epsilon \rightarrow 0$. Then we have

$$
\int_{B_{\frac{d}{\epsilon}}(0)}\left|x_{\alpha}+t_{\alpha}\right|^{m-2}\left(x_{\alpha}+t_{\alpha}\right)\left(U^{i}(x)\right)^{2}=0
$$

where $t_{\alpha}$ is the $\alpha$ th component of $t$ for $\alpha=1,2,3$.

As $U^{i}(x)$ is radially symmetric decreasing, we get $t=0$. This yields (2.3). Then, combining (2.3) and (2.12) yields (2.4). The proof of Proposition 2.2 is complete.

\section{Local uniqueness results}

In this section, we prove the local uniqueness results. We use a contradiction argument as that of Cao, Li and Luo [2]. Assume $u_{\epsilon}^{(i)}=\sum_{j=1}^{k} U_{\epsilon, x_{\epsilon}^{j(i)}}^{j}+\varphi^{(i)}, i=1,2$, are two distinct solutions concentrating on $\left\{b_{1}, \cdots, b_{k}\right\} \subset \mathbf{R}^{3}$. Set

$$
\xi_{\epsilon}=\frac{u_{\epsilon}^{(1)}-u_{\epsilon}^{(2)}}{\left\|u_{\epsilon}^{(1)}-u_{\epsilon}^{(2)}\right\|_{L^{\infty}\left(\mathbf{R}^{3}\right)}}
$$

and

$$
\bar{\xi}_{\epsilon}(x)=\xi_{\epsilon}\left(\epsilon x+x_{\epsilon}^{i_{0}(1)}\right)
$$

for an arbitrary $i_{0} \in\{1, \cdots, k\}$. In the below, $i_{0}$ will be fixed always. It is clear that

$$
\left\|\bar{\xi}_{\epsilon}\right\|_{L^{\infty}\left(\mathbf{R}^{3}\right)}=1 \text {. }
$$

To obtain a contradiction, in the rest of this section, we prove

$$
\left\|\bar{\xi}_{\epsilon}\right\|_{L^{\infty}\left(\mathbf{R}^{3}\right)}=o(1) .
$$

To prove (3.1), we will first prove that $\left|\bar{\xi}_{\epsilon}\right| \rightarrow o(1)$ holds locally uniformly, and then prove that it holds at infinity. To this end, we will establish a series of results. First we have

Proposition 3.1. There holds

$$
\left\|\xi_{\epsilon}\right\|_{\epsilon}=O\left(\epsilon^{3 / 2}\right) .
$$

Proof. Since both $u_{\epsilon}^{(i)}, i=1,2$, are assumed to be solutions to Eq. (1.1), we obtain

$$
\begin{aligned}
& -\left(\epsilon^{2} a+\epsilon b \int\left|\nabla u_{\epsilon}^{(1)}\right|^{2}\right) \Delta \xi_{\epsilon}+V(x) \xi_{\epsilon} \\
& -\epsilon b\left(\int \nabla\left(u_{\epsilon}^{(1)}+u_{\epsilon}^{(2)}\right) \cdot \nabla \xi_{\epsilon}\right) \Delta u_{\epsilon}^{(2)}=C_{\epsilon}(x) \xi_{\epsilon}
\end{aligned}
$$

and

$$
\begin{aligned}
& -\left(\epsilon^{2} a+\epsilon b \int\left|\nabla u_{\epsilon}^{(2)}\right|^{2}\right) \Delta \xi_{\epsilon}+V(x) \xi_{\epsilon} \\
& -\epsilon b\left(\int \nabla\left(u_{\epsilon}^{(1)}+u_{\epsilon}^{(2)}\right) \cdot \nabla \xi_{\epsilon}\right) \Delta u_{\epsilon}^{(1)}=C_{\epsilon}(x) \xi_{\epsilon}
\end{aligned}
$$


where

$$
C_{\epsilon}(x)=p \int_{0}^{1}\left(t u_{\epsilon}^{(1)}(x)+(1-t) u_{\epsilon}^{(2)}(x)\right)^{p-1} .
$$

Adding (3.2) and (3.3) together gives

$$
\begin{aligned}
& -\left(2 \epsilon^{2} a+\epsilon b \int\left|\nabla u_{\epsilon}^{(1)}\right|^{2}+\left|\nabla u_{\epsilon}^{(2)}\right|^{2}\right) \Delta \xi_{\epsilon}+2 V(x) \xi_{\epsilon} \\
& -\epsilon b\left(\int \nabla\left(u_{\epsilon}^{(1)}+u_{\epsilon}^{(2)}\right) \cdot \nabla \xi_{\epsilon}\right) \Delta\left(u_{\epsilon}^{(1)}+u_{\epsilon}^{(2)}\right)=2 C_{\epsilon}(x) \xi_{\epsilon} .
\end{aligned}
$$

Multiply $\xi_{\epsilon}$ on both sides of (3.4) and integrate over $\mathbf{R}^{3}$. As the terms containing $b$ are positive, we can throw away them. So

$$
\left\|\xi_{\epsilon}\right\|_{\epsilon}^{2} \leq \int C_{\epsilon} \xi_{\epsilon}^{2} \mathrm{~d} x
$$

As $\left|\xi_{\epsilon}\right| \leq 1$, for $i=1,2$, direct computation gives

$$
\int\left(U_{\epsilon, x_{\epsilon}^{j(i)}}^{j}\right)^{p-1} \xi_{\epsilon}^{2} \leq C \epsilon^{3}
$$

and

$$
\begin{aligned}
\int\left(\varphi^{(i)}\right)^{p-1} \xi_{\epsilon}^{2} & \leq\left\|\varphi^{(i)}\right\|_{p+1}^{p-1}\left\|\xi_{\epsilon}\right\|_{p+1}^{2} \leq C\left(\epsilon^{\frac{3}{p+1}-\frac{3}{2}}\left\|\varphi^{(i)}\right\|_{\epsilon}\right)^{p-1}\left(\epsilon^{\frac{3}{p+1}-\frac{3}{2}}\left\|\xi_{\epsilon}\right\|_{\epsilon}\right)^{2} \\
& =o_{\epsilon}(1)\left\|\xi_{\epsilon}\right\|_{\epsilon}^{2}
\end{aligned}
$$

where we have used (2.7) and Proposition 2.1. Hence

$$
\begin{aligned}
\left\|\xi_{\epsilon}\right\|_{\epsilon}^{2} & \leq \int C_{\epsilon} \xi_{\epsilon}^{2} \mathrm{~d} x \leq \int C\left(\sum_{i, j=1}^{2}\left(U_{\epsilon, x_{\epsilon}^{j(i)}}^{j}\right)^{p-1}+\sum_{i=1}^{2}\left(\varphi^{(i)}\right)^{p-1}\right) \xi_{\epsilon}^{2} \mathrm{~d} x \\
& =O\left(\epsilon^{3}\right)+o_{\epsilon}(1)\left\|\xi_{\epsilon}\right\|_{\epsilon}^{2},
\end{aligned}
$$

which implies the desired estimate. The proof is complete.

Next we study the asymptotic behavior of $\bar{\xi}_{\epsilon}$.

Proposition 3.2. There exist $d_{\beta} \in \mathbf{R}, \beta=1,2,3$, such that (up to a subsequence)

$$
\bar{\xi}_{\epsilon} \rightarrow \sum_{\beta=1}^{3} d_{\beta} \frac{\partial U^{i_{0}}}{\partial x_{\beta}} \quad \text { in } C_{\mathrm{loc}}^{1}\left(\mathbf{R}^{3}\right)
$$

as $\epsilon \rightarrow 0$. Here $i_{0}$ is the index used in the definition of $\bar{\xi}_{\epsilon}$.

Proof. We will prove that the limiting function of $\bar{\xi}_{\epsilon}$ belongs to the kernel of a linear operator associated to $U^{i_{0}}$. It is straightforward to deduce from (3.2) that $\bar{\xi}_{\epsilon}$ solves

$$
\begin{aligned}
& -\left(a+\epsilon^{-1} b \int\left|\nabla u_{\epsilon}^{(1)}\right|^{2}\right) \Delta \bar{\xi}_{\epsilon}+V\left(\epsilon x+x_{\epsilon}^{i_{0}(1)}\right) \bar{\xi}_{\epsilon} \\
& -\epsilon^{-1} b\left(\int \nabla\left(u_{\epsilon}^{(1)}+u_{\epsilon}^{(2)}\right) \cdot \nabla \xi_{\epsilon}\right) \Delta u_{\epsilon}^{(2)}\left(\epsilon x+x_{\epsilon}^{i_{0}(1)}\right)=C_{\epsilon}\left(\epsilon x+x_{\epsilon}^{i_{0}(1)}\right) \bar{\xi}_{\epsilon} .
\end{aligned}
$$

Thus, in view of $\left\|\bar{\xi}_{\epsilon}\right\|_{L^{\infty}\left(\mathbf{R}^{3}\right)}=1$, the elliptic regularity theory implies that $\bar{\xi}_{\epsilon}$ is locally uniformly bounded with respect to $\epsilon$ in $C_{\text {loc }}^{1, \theta}\left(\mathbf{R}^{3}\right)$ for some $\theta \in(0,1)$. As a consequence, we assume (up to a subsequence) that

$$
\bar{\xi}_{\epsilon} \rightarrow \bar{\xi} \quad \text { in } C_{\text {loc }}^{1}\left(\mathbf{R}^{3}\right) \text {. }
$$


We claim that $\bar{\xi} \in \operatorname{Ker} \mathcal{L}^{i_{0}}$, that is,

$$
\begin{aligned}
& -\left(a+b \sum_{j=1}^{k} \int\left|\nabla U^{j}\right|^{2}\right) \Delta \bar{\xi}-2 b\left(\int \nabla U^{i_{0}} \cdot \nabla \bar{\xi}\right) \Delta U^{i_{0}}+V\left(b_{i_{0}}\right) \bar{\xi} \\
& =p\left(U^{i_{0}}\right)^{p-1} \bar{\xi} .
\end{aligned}
$$

Then by the fact that $U^{i_{0}}$ is nondegenerate, we have $\bar{\xi}=\sum_{\beta=1}^{3} d_{\beta} \frac{\partial U^{i_{0}}}{\partial x_{\beta}}$ for some $d_{\beta} \in \mathbf{R}(\beta=1,2,3)$, and thus Proposition 3.2 is proved.

To deduce (3.6), we only need to show that (3.6) is the limiting equation of Eq. (3.5). Observing that

$$
\begin{aligned}
& \epsilon^{-1} \int\left|\nabla u_{\epsilon}^{(1)}\right|^{2}=\epsilon^{-1} \int\left|\sum_{j=1}^{k} \nabla U^{j}\left(\frac{x-x_{\epsilon}^{j(1)}}{\epsilon}\right)+\nabla \varphi^{(1)}\right|^{2} \\
= & \epsilon^{-1} \int\left|\sum_{j=1}^{k} \nabla U^{j}\left(\frac{x-x_{\epsilon}^{j(1)}}{\epsilon}\right)\right|^{2}+2 \epsilon^{-1} \int \sum_{j=1}^{k} \nabla U^{j}\left(\frac{x-x_{\epsilon}^{j(1)}}{\epsilon}\right) \cdot \nabla \varphi^{(1)}+\epsilon^{-1} \int\left|\nabla \varphi^{(1)}\right|^{2} \\
= & \epsilon^{-1} \int \sum_{j=1}^{k}\left|\nabla U^{j}\left(\frac{x-x_{\epsilon}^{j(1)}}{\epsilon}\right)\right|^{2} \\
& +\epsilon^{-1} \sum_{i \neq j} \int \nabla U^{j}\left(\frac{x-x_{\epsilon}^{j(1)}}{\epsilon}\right) \cdot \nabla U^{i}\left(\frac{x-x_{\epsilon}^{i(1)}}{\epsilon}\right)+O\left(\epsilon^{-\frac{3}{2}}\|\varphi\|_{\epsilon}\right) \\
= & \int \sum_{j=1}^{k}\left|\nabla U^{j}\right|^{2}+\sum_{i \neq j} \int \nabla U^{j}(z) \cdot \nabla U^{i}\left(z+\frac{x_{\epsilon}^{j(1)}-x_{\epsilon}^{i(1)}}{\epsilon}\right) d z+O\left(\epsilon^{-\frac{3}{2}}\|\varphi\|_{\epsilon}\right) \\
= & \int \sum_{j=1}^{k}\left|\nabla U^{j}\right|^{2}+O\left(e^{-\frac{\gamma}{\epsilon}}\right)+O\left(\epsilon^{m}\right),
\end{aligned}
$$

where we have used (2.5), (2.6) and (2.4), so

$$
\epsilon^{-1} \int\left|\nabla u_{\epsilon}^{(1)}\right|^{2}-\int \sum_{j=1}^{k}\left|\nabla U^{j}\right|^{2}=O\left(e^{-\frac{\gamma}{\epsilon}}\right)+O\left(\epsilon^{m}\right) \rightarrow 0
$$

as $\epsilon \rightarrow 0$. For convenience, we introduce

$$
\bar{u}_{\epsilon}^{(i)}(x)=u_{\epsilon}^{(i)}\left(\epsilon x+x_{\epsilon}^{i_{0}(1)}\right) \quad \text { and } \quad \bar{\varphi}_{\epsilon}^{(i)}=\varphi_{\epsilon}^{(i)}\left(\epsilon x+x_{\epsilon}^{i_{0}(1)}\right)
$$

for $i=1,2$. Similarly, we have

$$
\begin{aligned}
\epsilon^{-1} \int \nabla\left(u_{\epsilon}^{(1)}+u_{\epsilon}^{(2)}\right) \cdot \nabla \xi_{\epsilon}-2 \int \nabla U^{i_{0}} \cdot \nabla \bar{\xi}_{\epsilon} \\
=\int\left(\nabla \bar{u}_{\epsilon}^{(1)}+\nabla \bar{u}_{\epsilon}^{(2)}-2 \nabla U^{i_{0}}\right) \cdot \nabla \bar{\xi}_{\epsilon} \\
=\int\left(\sum_{j \neq i_{0}} \nabla U^{j}\left(z+\frac{x_{\epsilon}^{i_{0}(1)}-x_{\epsilon}^{j(1)}}{\epsilon}\right)+\sum_{j \neq i_{0}} \nabla U^{j}\left(z+\frac{x_{\epsilon}^{i_{0}(1)}-x_{\epsilon}^{j(2)}}{\epsilon}\right)\right. \\
\left.\quad+\nabla U^{i_{0}}(z)+\nabla U^{i_{0}}\left(z+\frac{x_{\epsilon}^{i_{0}(1)}-x_{\epsilon}^{i_{0}(2)}}{\epsilon}\right)-2 \nabla U^{i_{0}}(z)\right) \cdot \nabla \bar{\xi}_{\epsilon}+\int\left(\nabla \bar{\varphi}^{(1)}+\nabla \bar{\varphi}^{(2)}\right) \cdot \nabla \bar{\xi}_{\epsilon} \\
=O\left(e^{-\frac{\gamma}{\epsilon}}+\epsilon^{m-\frac{1}{2}}\right)\left\|\bar{\xi}_{\epsilon}\right\|_{\epsilon} \rightarrow 0
\end{aligned}
$$


as $\epsilon \rightarrow 0$, and that, for any $\Phi \in C_{0}^{\infty}\left(\mathbf{R}^{3}\right)$,

$$
\begin{aligned}
& \int \nabla\left(u_{\epsilon}^{(2)}\left(\epsilon x+x_{\epsilon}^{i_{0}(1)}\right)-U^{i_{0}}\right) \cdot \nabla \Phi \\
& =\int \nabla\left(U^{i_{0}}\left(z+\frac{x_{\epsilon}^{i_{0}(1)}-x_{\epsilon}^{i_{0}(2)}}{\epsilon}\right)+\sum_{j \neq i_{0}} U^{j}\left(z+\frac{x_{\epsilon}^{i_{0}(1)}-x_{\epsilon}^{j(2)}}{\epsilon}\right)-U^{i_{0}}(z)\right) \cdot \nabla \Phi \\
& \quad+\int \nabla \bar{\varphi}^{(2)} \cdot \nabla \Phi=O\left(e^{-\frac{\gamma}{\epsilon}}+\epsilon^{m-\frac{1}{2}}\right)\|\Phi\|_{\epsilon} \rightarrow 0 .
\end{aligned}
$$

Here, we have used (2.3), which implies

$$
\frac{x_{\epsilon}^{i_{0}(1)}-x_{\epsilon}^{i_{0}(2)}}{\epsilon}=o(1)
$$

and used

$$
\frac{x_{\epsilon}^{i_{0}(1)}-x_{\epsilon}^{j(i)}}{\epsilon} \rightarrow \infty \text { for } i=1,2 \text { and } j \neq i_{0} .
$$

as $\epsilon \rightarrow 0$. Combining the above two formulas and $\bar{\xi}_{\epsilon} \rightarrow \bar{\xi}$ in $C_{\text {loc }}^{1}\left(\mathbf{R}^{3}\right)$, we conclude that

$$
\begin{aligned}
& \epsilon^{-1} b\left(\int \nabla\left(u_{\epsilon}^{(1)}+u_{\epsilon}^{(2)}\right) \cdot \nabla \xi_{\epsilon}\right) \Delta u_{\epsilon}^{(2)}\left(\epsilon x+x_{\epsilon}^{i_{0}(1)}\right) \\
& \rightarrow 2 b\left(\int \nabla U^{i_{0}} \cdot \nabla \bar{\xi}\right) \Delta U^{i_{0}}
\end{aligned}
$$

in $H^{-1}\left(\mathbf{R}^{3}\right)$. Since $V\left(\epsilon x+x_{\epsilon}^{i_{0}(1)}\right) \rightarrow V\left(b_{i_{0}}\right)$ holds locally uniformly, we have

$$
V\left(\epsilon x+x_{\epsilon}^{i_{0}(1)}\right) \bar{\xi}_{\epsilon} \rightarrow V\left(b_{i_{0}}\right) \bar{\xi}
$$

Also, similar to Cao et. al. [2, proof of (3.11)], we have for any $\Phi \in C_{0}^{\infty}\left(\mathbf{R}^{3}\right)$,

$$
\int C_{\epsilon}\left(\epsilon x+x_{\epsilon}^{i_{0}(1)}\right) \bar{\xi}_{\epsilon} \Phi \rightarrow \int p\left(U^{i_{0}}\right)^{p-1} \bar{\xi} \Phi
$$

Finally, combining (3.7) (3.8) (3.9), we obtain (3.6). The proof is complete.

Now we prove

Proposition 3.3. Let $d_{\beta}$ be defined as in Proposition 3.2. Then

$$
d_{\beta}=0 \text { for } \beta=1,2,3 .
$$

Proof. We will combine Proposition 2.2 and the Pohozaev-type identity (2.8) to prove that

$$
d_{\beta} \int\left|x_{\alpha}\right|^{m-2} x_{\alpha} U^{i_{0}}(x) \frac{\partial U^{i_{0}}}{\partial x_{\beta}}=0 .
$$

Applying (2.8) to $u_{\epsilon}^{(1)}$ and $u_{\epsilon}^{(2)}$ with $\Omega=B_{d}\left(x_{\epsilon}^{i_{0}(1)}\right)$, where $d$ is chosen in the same way as that of Proposition 2.2, which combine with (2.4), (2.13) and Proposition 3.1 implies

$$
\int_{\partial B_{d}\left(x_{\epsilon}^{i_{0}(1)}\right)}\left(\epsilon^{2}\left|\nabla \varphi^{(i)}\right|^{2}+V(x)\left|\varphi^{(i)}\right|^{2}\right)=O\left(\left\|\varphi^{(i)}\right\|_{\epsilon}^{2}\right)=O\left(\epsilon^{3+2 m}\right)
$$

for $i=1,2$ and

$$
\int_{\partial B_{d}\left(x_{\epsilon}^{i_{0}(1)}\right)}\left(\epsilon^{2}\left|\nabla \xi_{\epsilon}\right|^{2}+V(x)\left|\xi_{\epsilon}\right|^{2}\right)=O\left(\epsilon^{3}\right)
$$


We obtain

$$
\begin{aligned}
& \int_{B_{d}\left(x_{\epsilon}^{i_{0}(1)}\right)} \frac{\partial V}{\partial x_{\alpha}}\left(u_{\epsilon}^{(1)}+u_{\epsilon}^{(2)}\right) \xi_{\epsilon} \\
& =\left(\epsilon^{2} a+\epsilon b \int\left|\nabla u_{\epsilon}^{(1)}\right|^{2}\right) \int_{\partial B_{d}\left(y_{\epsilon}^{(1)}\right)}\left(\nabla u_{\epsilon}^{(1)}+\nabla u_{\epsilon}^{(2)}\right) \cdot \nabla \xi_{\epsilon} \nu_{\alpha} \\
& \quad+\left(\epsilon^{2} a+\epsilon b \int\left|\nabla u_{\epsilon}^{(2)}\right|^{2}\right) \int_{\partial B_{d}\left(y_{\epsilon}^{(1)}\right)}\left(\frac{\partial \xi_{\epsilon}}{\partial x_{\alpha}} \frac{\partial u_{\epsilon}^{(2)}}{\partial \nu}-\frac{\partial \xi_{\epsilon}}{\partial \nu} \frac{\partial u_{\epsilon}^{(1)}}{\partial x_{\alpha}}\right) \\
& +\epsilon b\left(\int \nabla\left(u_{\epsilon}^{(1)}+u_{\epsilon}^{(2)}\right) \cdot \nabla \xi_{\epsilon}\right) \int_{\partial B_{d}\left(y_{\epsilon}^{(1)}\right)}\left(\left|\nabla u_{\epsilon}^{(2)}\right|^{2} \nu_{\alpha}-2 \partial_{\nu} u_{\epsilon}^{(1)} \partial_{\alpha} u_{\epsilon}^{(1)}\right) \\
& \quad+\int_{\partial B_{d}\left(x_{\epsilon}^{i_{0}(1)}\right)} V(x)\left(u_{\epsilon}^{(1)}+u_{\epsilon}^{(2)}\right) \xi_{\epsilon} \nu_{\alpha}-2 \int_{\partial B_{d}\left(x_{\epsilon}^{i_{0}(1)}\right)} A_{\epsilon} \xi_{\epsilon} \nu_{\alpha},
\end{aligned}
$$

where $1 \leq \alpha \leq 3$ and

$$
A_{\epsilon}=\int_{0}^{1}\left(t u_{\epsilon}^{(1)}+(1-t) u_{\epsilon}^{(2)}\right)^{p}
$$

We estimate (3.13) term by term. By (2.16), (3.11) and (3.12), we have

$$
\begin{aligned}
& \left(\epsilon^{2} a+\epsilon b \int\left|\nabla u_{\epsilon}^{(1)}\right|^{2}\right) \int_{\partial B_{d}\left(y_{\epsilon}^{(1)}\right)}\left|\nabla\left(u_{\epsilon}^{(1)}+u_{\epsilon}^{(2)}\right) \cdot \nabla \xi_{\epsilon} \nu_{\alpha}\right| \\
& \leq C\left(\epsilon^{2} \int_{\partial B_{d}\left(y_{\epsilon}^{(1)}\right)}\left|\nabla u_{\epsilon}^{(1)}\right|^{2}\right)^{\frac{1}{2}}\left(\epsilon^{2} \int_{\partial B_{d}\left(y_{\epsilon}^{(1)}\right)}\left|\nabla \xi_{\epsilon}\right|^{2}\right)^{\frac{1}{2}} \\
& =O\left(\epsilon^{\gamma}+\left\|\varphi_{\epsilon}\right\|_{\epsilon}\right) O\left(\epsilon^{\frac{3}{2}}\right)=O\left(\epsilon^{3+m}\right)
\end{aligned}
$$

by choosing $\gamma$ sufficiently large. Similarly, we obtain

$$
\left(\epsilon^{2} a+\epsilon b \int\left|\nabla u_{\epsilon}^{(2)}\right|^{2}\right) \int_{\partial B_{d}\left(y_{\epsilon}^{(1)}\right)}\left(\frac{\partial \xi_{\epsilon}}{\partial x_{\alpha}} \frac{\partial u_{\epsilon}^{(2)}}{\partial \nu}-\frac{\partial \xi_{\epsilon}}{\partial \nu} \frac{\partial u_{\epsilon}^{(1)}}{\partial x_{\alpha}}\right)=O\left(\epsilon^{3+m}\right)
$$

and

$$
\epsilon b\left(\int \nabla\left(u_{\epsilon}^{(1)}+u_{\epsilon}^{(2)}\right) \cdot \nabla \xi_{\epsilon}\right) \int_{\partial B_{d}\left(y_{\epsilon}^{(1)}\right)}\left(\left|\nabla u_{\epsilon}^{(2)}\right|^{2} \nu_{\alpha}-2 \partial_{\nu} u_{\epsilon}^{(1)} \partial_{i} u_{\epsilon}^{(1)}\right)=O\left(\epsilon^{3+m}\right) .
$$

By (3.11) and (3.12), we have

$$
\begin{aligned}
& \int_{\partial B_{d}\left(x_{\epsilon}^{i_{0}(1)}\right)} V(x)\left(u_{\epsilon}^{(1)}+u_{\epsilon}^{(2)}\right) \xi_{\epsilon} \nu_{\alpha} \\
& \leq C \sum_{i=1}^{2}\left(\int_{\partial B_{d}\left(x_{\epsilon}^{i_{0}(1)}\right)}\left|u_{\epsilon}^{(i)}\right|^{2}\right)^{\frac{1}{2}}\left(\int_{\partial B_{d}\left(x_{\epsilon}^{i_{0}(1)}\right)}\left|\xi_{\epsilon}\right|^{2}\right)^{\frac{1}{2}} \\
& =O\left(\epsilon^{\frac{3}{2}+m} \cdot \epsilon^{\frac{3}{2}}\right)=O\left(\epsilon^{3+m}\right) .
\end{aligned}
$$

As to $A_{\epsilon}$, we have

$$
A_{\epsilon}=O\left(\epsilon^{\gamma}+\sum_{i=1}^{2}\left|\varphi^{(i)}\right|^{p}\right) \quad \text { on } \partial B_{d}\left(x_{\epsilon}^{i_{0}(1)}\right)
$$


for any given $\gamma>0$, since $U^{j}, j=1, \cdots, k$, decay exponentially at infinity. Hence we can deduce that

$$
\begin{aligned}
\int_{\partial B_{d}\left(x_{\epsilon}^{i_{0}(1)}\right)} A_{\epsilon} \xi_{\epsilon} \nu_{\alpha} & \leq C \epsilon^{\gamma}\left(\int_{\partial B_{d}\left(x_{\epsilon}^{i_{0}(1)}\right)}\left|\xi_{\epsilon}\right|^{2}\right)^{\frac{1}{2}}+C \sum_{i=1}^{2}\left(\int_{\partial B_{d}\left(x_{\epsilon}^{i_{0}(1)}\right)}\left|\varphi^{(i)}\right|^{p}\right)\left|\xi_{\epsilon}\right| \\
& \leq C \epsilon^{\gamma+\frac{3}{2}}+O\left(\sum_{i=1}^{2}\left(\epsilon^{\frac{3}{p+1}-\frac{3}{2}}\left\|\varphi^{(i)}\right\|_{\epsilon}\right)^{p}\left(\epsilon^{\frac{3}{p+1}-\frac{3}{2}}\left\|\xi_{\epsilon}\right\|_{\epsilon}\right)\right) \\
& =O\left(\epsilon^{3+p m}\right),
\end{aligned}
$$

by choosing $\gamma>\frac{3}{2}+p m$.

Hence by the above estimate, there holds

$$
\int_{B_{d}\left(x_{\epsilon}^{i_{0}(1)}\right)} \frac{\partial V}{\partial x_{\alpha}}\left(u_{\epsilon}^{(1)}+u_{\epsilon}^{(2)}\right) \xi_{\epsilon}=O\left(\epsilon^{3+m}\right) .
$$

Next we estimate the left hand side of (3.14). By the assumption (V2), we have

$$
\begin{aligned}
& \int_{B_{d}\left(x_{\epsilon}^{i_{0}(1)}\right)} \frac{\partial V}{\partial x_{\alpha}}\left(u_{\epsilon}^{(1)}+u_{\epsilon}^{(2)}\right) \xi_{\epsilon} \\
& =m c_{i_{0}, \alpha} \int_{B_{d}\left(x_{\epsilon}^{i_{0}(1)}\right)}\left|x_{\alpha}-b_{i_{0}, \alpha}\right|^{m-2}\left(x_{\alpha}-b_{i_{0}, \alpha}\right)\left(u_{\epsilon}^{(1)}+u_{\epsilon}^{(2)}\right) \xi_{\epsilon} \\
& \quad+O\left(\int_{B_{d}\left(x_{\epsilon}^{i_{0}(1)}\right)}\left|x-b_{i_{0}}\right|^{m}\left(u_{\epsilon}^{(1)}+u_{\epsilon}^{(2)}\right) \xi_{\epsilon}\right)=: I_{1}+I_{2} .
\end{aligned}
$$

Note that

$$
I_{2}=O\left(\epsilon^{3+m} \int_{B_{\frac{d}{\epsilon}}(0)}\left|x+\frac{x_{\epsilon}^{i_{0}(1)}-b_{i_{0}}}{\epsilon}\right|^{m}\left(U^{i_{0}}+U^{i_{0}}\left(x+\frac{x_{\epsilon}^{i_{0}(1)}-x_{\epsilon}^{i_{0}(2)}}{\epsilon}\right)+e\right) \bar{\xi}_{\epsilon}(x)\right),
$$

where

$$
e=\sum_{i=1}^{2} \sum_{j \neq i_{0}} U^{j}\left(x+\frac{x_{\epsilon}^{i_{0}(1)}-x^{j(i)}}{\epsilon}\right)+\sum_{i=1}^{2} \varphi_{\epsilon}^{i}\left(\epsilon x+x_{\epsilon}^{i_{0}(1)}\right) .
$$

Using the estimates (2.3) and (2.4) and the exponential decay of $U^{j}(j=1, \ldots, k)$, and the fact that $\left\|\bar{\xi}_{\epsilon}\right\|_{\infty}=1$ we deduce that

$$
I_{2}=O\left(\epsilon^{3+m}\right) \text {. }
$$

Combining the above estimate with (3.14) yields

$$
I_{1}=O\left(\epsilon^{3+m}\right) \text {. }
$$

By the same arguments as that of $I_{2}$, and using the assumption that $c_{i, \alpha} \neq 0$ for every $i, \alpha$ and using Proposition 3.2, we deduce

$$
\begin{aligned}
& d_{\alpha} \int_{B_{\frac{d}{\epsilon}}(0)}\left|x_{\alpha}+o_{\epsilon}(1)\right|^{m-2}\left(x_{\alpha}+o_{\epsilon}(1)\right)\left(U^{i_{0}}+U^{i_{0}}\left(x+\frac{x_{\epsilon}^{i_{0}(1)}-x_{\epsilon}^{i_{0}(2)}}{\epsilon}\right)+e\right) \bar{\xi}_{\epsilon}(x) \\
= & O(\epsilon)
\end{aligned}
$$

which implies

$$
\sum_{\beta=1}^{3} d_{\beta} \int\left|x_{\alpha}\right|^{m-1} x_{\alpha} U^{i_{0}}(x) \frac{\partial U^{i_{0}}}{\partial x_{\beta}}(x)=0 .
$$

Thus $d_{\alpha}=0$ for $\alpha=1,2,3$. The proof is complete. 
Now we can complete the proof of Theorem 1.2.

Proof of Theorem 1.2. Propositions 3.2 and 3.3 imply that

$$
\left\|\bar{\xi}_{\epsilon}\right\|_{L^{\infty}\left(B_{R}(0)\right)}=o_{\epsilon}(1)
$$

so we have

$$
\left\|\xi_{\epsilon}\right\|_{L^{\infty}\left(\bigcup_{i=1}^{k} B_{R \epsilon}\left(x_{\epsilon}^{i(1)}\right)\right)}=o_{\epsilon}(1) .
$$

In the domain $\mathbf{R}^{3} \backslash \bigcup_{i=1}^{k} B_{R \epsilon}\left(x_{\epsilon}^{i(1)}\right)$, we can apply the same argument as that of [2, Proposition 3.5] to conclude that

$$
\left\|\xi_{\epsilon}\right\|_{L^{\infty}\left(\mathbf{R}^{3} \backslash \bigcup_{i=1}^{k} B_{R \epsilon}\left(x_{\epsilon}^{i(1)}\right)\right)}=o_{\epsilon}(1) .
$$

Thus $\left\|\xi_{\epsilon}\right\|_{L^{\infty}\left(\mathbf{R}^{3}\right)}=o_{\epsilon}(1)$ holds, which is in contradiction with $\left\|\xi_{\epsilon}\right\|_{L^{\infty}\left(\mathbf{R}^{3}\right)}=1$. The proof is complete.

Acknowledgement. The authors would like to thank the anonymous referees for their valuable comments which helped to improve the manuscript.

\section{References}

[1] CAO, D., and H.P. HeInZ: Uniqueness of positive multi-lump bound states of nonlinear Schrödinger equations. - Math. Z. 243, 2003, 599-642.

[2] CAO, D., S. Li, and P. LuO: Uniqueness of positive bound states with multi-bump for nonlinear Schrödinger equations. - Calc. Var. Partial Differential Equations 54, 2015, 4037-4063.

[3] CaO, D., E.S. Noussair, and S. YAn: Solutions with multiple peaks for nonlinear elliptic equations. - Proc. Royal Soc. Edinburgh 129, 1999, 235-264.

[4] Del Pino, M., and P. L. Felmer: Local mountain passes for semilinear elliptic problems in unbounded domains. - Calc. Var. Partial Differential Equations 4, 1996, 121-137.

[5] Del Pino, M., and P. L. Felmer: Multi-peak bound states for nonlinear Schrödinger equations. - Ann. Inst. H. Poincaré Anal. Non Linéaire 15, 1998, 127-149.

[6] Deng, Y., C. Lin, and S. YAn: On the prescribed scalar curvature problem in $\mathbf{R}^{N}$, local uniqueness and periodicity. - J. Math. Pures Appl. (9) 104, 2015, 1013-1044.

[7] Figueiredo, G. M., and J. R. SAntos Júnior: Multiplicity and concentration behavior of positive solutions for a Schrödinger-Kirchhoff type problem via penalization method. - ESAIM Control Optim. Calc. Var. 20, 2014, 389-415.

[8] Flower, A., and A. Weinstein: Nonspeading wave packets for the cubic Schrödinger equation with a bounded potential. - J. Funct. Anal. 69, 1986, 397-408.

[9] Gidas, B., W. Ni, and L. NirenberG: Symmetry and related properties via the maximum principle. - Commun. Math. Phys. 68, 1979, 209-243.

[10] Glangetas, L.: Uniqueness of positive solutions of a nonlinear equation involving the critical exponent. - Nonlinear Anal. 20, 1993, 115-178.

[11] Grossi, M.: On the number of single-peak solutions of the nonlinear Schrödinger equation. Ann. Inst. H. Poincaré Anal. Non Linéaire 19, 2002, 261-280.

[12] GuI, C.: Existence of multi-bump solutions for nonlinear Schrödinger equations via variational method. - Comm. Partial Differential Equations 21, 1996, 787-820.

[13] Guo, Y., J. Nie, M. Niu, and Z. TANG: Local uniqueness and periodicity for the prescribed scalar curvature problem of fractional operator in $\mathbf{R}^{N}$. - Calc. Var. Partial Differential Equations 56:4, 2017, Art. 118, 1-41.

[14] Guo, Y., S. Peng, and S. YAn: Local uniqueness and periodicity induced by concentration. - Proc. Lond. Math. Soc. (3) 114, 2017, 1005-1043. 
[15] He, X., and W. Zou: Existence and concentration behavior of positive solutions for a Kirchhoff equation in $\mathbf{R}^{3}$. - J. Differential Equations 252, 2012, 1813-1834.

[16] HE, Y.: Concentrating bounded states for a class of singularly perturbed Kirchhoff type equations with a general nonlinearity. - J. Differential Equations 261, 2016, 6178-6220.

[17] He, Y., and G. LI: Standing waves for a class of Kirchhoff type problems in $\mathbf{R}^{3}$ involving critical Sobolev exponents. - Calc. Var. Partial Differential Equations 54, 2015, 3067-3106.

[18] He, Y., G. LI, and S. Peng: Concentrating bound states for Kirchhoff type problems in $\mathbf{R}^{3}$ involving critical Sobolev exponents. - Adv. Nonlinear Stud. 14, 2014, 483-510.

[19] Hu, T., and W. ShuAs: Multi-peak solutions to Kirchhoff equations in $\mathbf{R}^{3}$ with general nonlinearity. - J. Differential Equations 265, 2018, 3587-3617.

[20] Kwong, M. K.: Uniqueness of positive solutions of $\Delta u-u+u^{p}=0$ in $\mathbf{R}^{n}$. - Arch. Rational Mech. Anal. 105, 1989, 243-266.

[21] Li, G., P. Luo, S. Peng, C. Wang, and C.-L. Xiang: Uniqueness and Nondegeneracy of positive solutions to Kirchhoff equations and its applications in singular perturbation problems. - Preprint, arXiv:1703.05459 [math.AP].

[22] Luo, P., S. Peng, C. Wang, and C.-L. XIAng: Multi-peak positive solutions to a class of Kirchhoff equations. - Proc. Roy. Soc. Edinburgh Sect. A 149, 2019, 1097-1122.

[23] Он, Y. G.: Existence of semiclassical bound states of nonlinear Schrödinger equations with potentials of class $(V)_{a}$. - Comm. Partial Differential Equations 13, 1988, 1499-1519.

[24] Он, Y.G.: On positive multi-lump bound states of nonlinear Schrödinger equations under multiple well potential. - Commun. Math. Phys. 131, 1990, 223-253.

[25] Rabinowitz, P. H.: On a class of nonlinear Schrödinger equations. - Z. Angew. Math. Phys. 43, 1992, 270-291.

[26] Wang, J., L. Tian, J. Xu, and F. Zhang: Multiplicity and concentration of positive solutions for a Kirchhoff type problem with critical growth. - J. Differential Equations 253, 2012, 23142351.

Received 26 October 2018 • Accepted 28 February 2019 